\title{
Pérdida de glutatión desde pulmones de conejo en solución de preservación para trasplante. Efectos clínicos y funcionales a corto plazo
}

\author{
MARÍA E. SOLOVERA R.*, JUAN E. CHEYRE F.*, ANDREA VECCHIOLA C.*, \\ PILAR CELIS L.*, PABLO SANDOVAL A.* y GISELLA BORZONE T.*
}

\section{Loss of glutathione from rabbit lungs in transplant preservation solution}

Introduction: Ischemia-reperfusion injury causes morbidity and mortality in lung transplant patients. It is unknown if the usual lung preservation solution favors antioxidant deficiency and thus, increased oxidative stress in the receptor. Objective: To evaluate if there is any loss of glutathione from rabbit lungs to the preservation solution. Results: We found a decrease in lung total glutathione content, without an increase in the oxidized form of this antioxidant. At the same time, we were able to measure increasing levels of glutathione in the preservation solution from 30 min on. Conclusions: There is loss of glutathione from the lung to the preservation solution that is not mediated by glutathione oxidation and likely due to passage of the reduced form along the concentration gradient, rendering the tissue vulnerable to oxidative stress once in the receptor.

Key words: Transplant, glutathione, oxidative stress, ischemia-reperfusion.

\section{Resumen}

El daño por isquemia-reperfusión es causa de morbimortalidad en pacientes con trasplante pulmonar. Se desconoce si la solución de preservación habitual del pulmón puede contribuir a la deficiencia de antioxidantes, favoreciendo el estrés oxidativo en el receptor. Objetivo: Evaluar si existe pérdida de glutatión desde pulmones de conejo a la solución de preservación para trasplante. Resultados: Encontramos una disminución en el contenido de glutatión total del pulmón, sin aumento en el contenido de glutatión oxidado. Esto se asoció a la aparición y aumento sostenido de glutatión en la solución de preservación desde los 30 min. Conclusiones: Existe salida de glutatión desde el pulmón no mediada por oxidación de éste y posiblemente favorecida por el gradiente de concentración de glutatión reducido. Esta pérdida deja al tejido vulnerable frente a las condiciones de estrés oxidativo en el receptor.

Palabras clave: Trasplante, glutatión, estrés oxidativo, isquemia-reperfusión, solución de preservación.

\section{Introducción}

El trasplante pulmonar constituye el único tratamiento definitivo para pacientes que sufren de insuficiencia respiratoria crónica terminal ${ }^{1}$. Si bien este procedimiento ofrece una mejoría significativa tanto de la calidad de vida (a los 5 años, $80 \%$ de los pacientes no presenta limitaciones para sus actividades diarias), como de la sobre- vida (sobrevida a 5 años entre 44-55\% según la enfermedad de base $)^{2}$, se acompaña de significativa morbilidad y mortalidad (mortalidad a 90 días de $9-15 \%)^{3}$.

Un $10-30 \%$ de la morbilidad peri-operatoria precoz se debe al Síndrome de Disfunción Primaria del Injerto ${ }^{4,5}$, debido en gran parte a fenómenos de isquemia-reperfusión. La repercusión clínica de este síndrome, que se manifiesta den-

* Sección de Cirugía de Tórax, Departamento de Enfermedades Respiratorias y Centro de Investigaciones Médicas. Pontificia Universidad Católica de Chile.

Financiado por el Programa Inicio en Investigación. Vicerrectoría Adjunta de Investigación y Doctorado. Pontificia Universidad Católica de Chile. 
tro de las primeras 72 horas después del trasplante, va desde la aparición de hipoxemia asociada a algunos infiltrados en la radiografía de tórax, hasta el Síndrome de Distress Respiratorio Agudo severo que puede requerir de oxigenación por membrana extracorpórea ${ }^{6}$, con elevada mortalidad del receptor. Se ha planteado que este síndrome se produce como consecuencia del desbalance entre oxidantes y antioxidantes, el cual se establece al aumentar bruscamente la producción de radicales libres durante la reperfusión del órgano en el receptor ${ }^{7,8}$. Sin embargo, existen evidencias en otros tejidos que sugieren que este desbalance puede estar agravado por la pérdida de reserva antioxidante del tejido trasplantado, tanto por factores dependientes del manejo clínico del donante ${ }^{8}$ como por las características del método de preservación del órgano antes del trasplante ${ }^{8}$.

En relación con el método de preservación, se ha encontrado disminución de la concentración de glutatión, uno de los principales antioxidantes celulares, en tejidos como el riñón, el hígado y el corazón, cuando son mantenidos por tiempo prolongado en soluciones de preservación para trasplante ${ }^{9}$. De igual forma, la concentración de glutatión disminuye en secciones del músculo diafragma de la rata cuando éstas se mantienen en solución de Krebs ${ }^{10}$.

Se desconoce si en el pulmón ocurre un fenómeno similar, que pudiese agravar el desbalance entre oxidantes y antioxidantes que se sabe ocurre en el receptor.

Nuestro objetivo fue estudiar si existe pérdida de glutatión del pulmón hacia la solución de preservación que no contiene glutatión, utilizando un modelo experimental de pulmón de conejo en condiciones similares a las del procuramiento para trasplante de pulmón en seres humanos.

\section{Material y Método}

Implementamos en conejos, un modelo experimental de extracción de pulmones en condiciones de isquemia fría, similares a las del procuramiento para trasplante de pulmón humano. El estudio fue aprobado por el Comité de Ética de la Facultad de Medicina de la Pontificia Universidad Católica de Chile.

Modelo experimental: Se utilizaron 6 conejos macho de 1,9 $\pm 0,44 \mathrm{~kg}$, los que fueron sedados con acepromazina (Pacifor ${ }^{\circledR}$ ) en dosis de $0,5-1,0 \mathrm{mg} / \mathrm{kg}$ para el traslado desde el
Bioterio hasta la sala de cirugía y luego anestesiados con Xilazina (Rompun $\left.{ }^{\circledR}\right) \quad 0,5 \mathrm{mg} / \mathrm{kg}$ y ketamina $0,5 \mathrm{mg} / \mathrm{kg}$ por vía intramuscular. Posteriormente se instaló una vía venosa con una infusión de suero fisiológico para la administración de pequeños bolos de Xilazina y Ketamina, con el objetivo de mantener una adecuada profundidad de la anestesia durante todo el procedimiento.

Una vez anestesiados, los conejos fueron sometidos a una variación de la técnica descrita por Shimoyama ${ }^{11}$. Fueron ventilados a través de una traqueostomía quirúrgica, con aire ambiental, un volumen corriente de 150-200 ml, una frecuencia respiratoria de 40/minuto y una presión de final de espiración (PEEP) de $5 \mathrm{~cm}$ $\mathrm{H}_{2} \mathrm{O}$. Luego se realizó una esternotomía media, canulando la arteria pulmonar a través del ventrículo derecho para perfundir la circulación pulmonar con solución de preservación Perfadex ${ }^{\circledR}$ (o LPD-low-potassium/Dextran, Vitrolife, Gothenburg, Sweden) a $4{ }^{\circ} \mathrm{C}$ desde una altura de $25 \mathrm{~cm}$, a fin de evitar el edema pulmonar. Esta solución es baja en $\mathrm{K}^{+}$, lo que conferiría protección a la célula endotelial. Además posee un coloide osmóticamente activo (Dextran 40), que actúa reteniendo agua en el espacio intravascular, con lo que se reduce la agregación eritrocitaria y plaquetaria. Si bien posee un bajo contenido de glucosa, éste es suficiente para mantener el metabolismo celular en condiciones de hipotermia ${ }^{12}$. Sin embargo, esta solución carece de los antioxidantes propios del líquido extracelular ${ }^{13}$ (Tabla 1). El drenaje venoso, para exanguinar el animal se realizó a través de una incisión en la aurícula izquierda y en la vena cava inferior. Posteriormente se realizó una

\section{Tabla 1. Composición de la solución LPD (low-potassium/dextran) ${ }^{6}$}

\begin{tabular}{lc}
\hline Composición & \\
\hline $\mathrm{Na}^{+}$ & 138 \\
$\mathrm{~K}^{+}$ & 6 \\
$\mathrm{Cl}^{-}$ & 142 \\
$\mathrm{Mg}^{++}$ & 0,8 \\
$\mathrm{SO}_{4}^{-2}$ & 0,8 \\
$\mathrm{PO}_{4}^{-3}$ & 0,8 \\
$\mathrm{Ca}^{++}$ & 0,3 \\
$\mathrm{HCO}_{3}^{-}$ & 1 \\
Dextran 40 & 50 \\
$\mathrm{Glucosa}^{-}$ & 0,9 \\
\hline
\end{tabular}

\# unidades en mmol/L salvo Dextran y glucosa que están expresadas en $\mathrm{g} / \mathrm{L}$. 
neumonectomía derecha y la incubación del pulmón en solución Perfadex ${ }^{\circledR}$ a $4{ }^{\circ} \mathrm{C}$. Se tomaron muestras de tejido pulmonar previo a la incubación y cada 30 min durante la incubación por un total de $2 \mathrm{~h}$, para la medición de la concentración pulmonar de glutatión (GSH) total y glutatión oxidado (GSSG). Además, a los mismos tiempos, se tomó muestras de la solución de incubación, para determinar la concentración de GSH total. Estos tiempos fueron utilizados ya que un estudio preliminar durante la implementación del modelo experimental, mostró que si bien es posible detectar muy precozmente la presencia de GSH en la solución, el contenido de GSH pulmonar no muestra una caída significativa antes de los $30 \mathrm{~min}$ (resultados no mostrados).

Contenido de glutatión total y oxidado: La determinación del contenido de GSH total y de GSSG, se realizó utilizando el método espectrofotométrico de Griffith ${ }^{14,15}$. Para la medición del contenido de GSSG se usó derivatización con vinilpiridina ${ }^{14}$. Los resultados se expresaron como nmol de equivalentes de GSH reducido por gramo de tejido húmedo y como nmol de equivalentes de GSH reducido por $\mathrm{ml}$ de solución de preservación.

Análisis estadístico: Se aplicó análisis de regresión no lineal y regresión lineal, utilizando GraphPad Prism ${ }^{\circledR}$ versión 4 (GraphPad Software Inc).

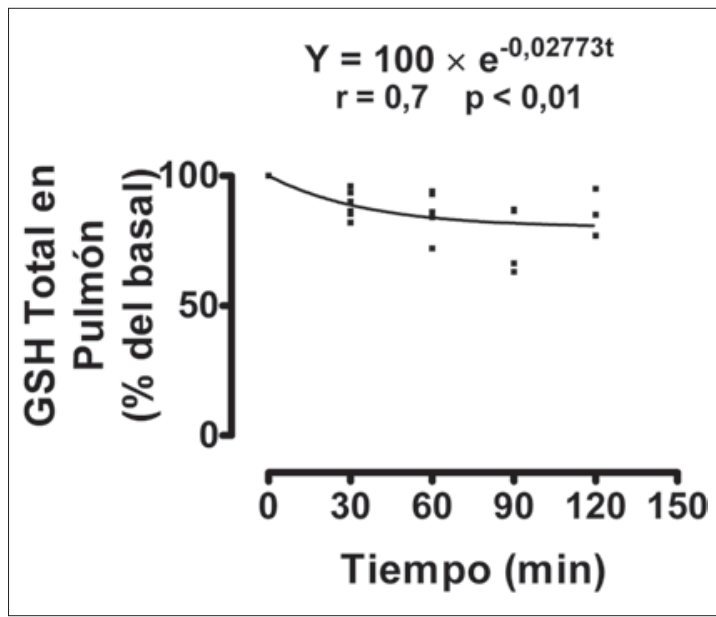

Figura 1. Concentración de glutatión total en el pulmón a distintos tiempos de incubación en la solución de preservación, expresada como porcentaje del valor basal para cada conejo. Es posible observar una caída en el contenido de GSH total que es más rápida al comienzo del período y que se ajusta a una curva exponencial de exponente negativo $(\mathrm{r}=0,7 ; \mathrm{p}<0,01)$.

\section{Resultados}

\section{Contenido basal de glutatión total y oxidado en el pulmón}

El contenido basal de glutatión total en el pulmón de conejo fue de $3.323 \pm 668 \mathrm{nmol} \mathrm{x} \mathrm{g}^{-1}$. Un $2,4 \pm 1,9 \%$ de éste correspondió a la forma oxidada del antioxidante.

\section{Contenido de glutatión total en pulmones a distintos tiempos de incubación en la solución de preservación para trasplante}

La Figura 1 muestra el contenido de GSH total del pulmón para cada conejo a distintos tiempos de incubación en la solución de preservación, expresado como porcentaje de su contenido basal (tiempo 0).

La caída en el contenido de GSH total en el tejido pulmonar es más acentuada al comienzo y tiende a ser menor en el tiempo, ajustándose a una función exponencial de exponente negativo $(\mathrm{r}=0,7 ; \mathrm{p}<0,01)$.

\section{Aparición de glutatión en la solución de preservación y aumento de su concentración durante el período de incubación}

La Figura 2 muestra que a los $30 \mathrm{~min}$ de incubación es posible detectar la presencia de glutatión en la solución de preservación de los pulmones en todos los conejos estudiados. El contenido de GSH total aumenta progresivamente, siendo este aumento mayor al comienzo del tiempo estudiado y ajustándose también a una curva de tipo exponencial $(r=0,75 ; p<0,001)$.

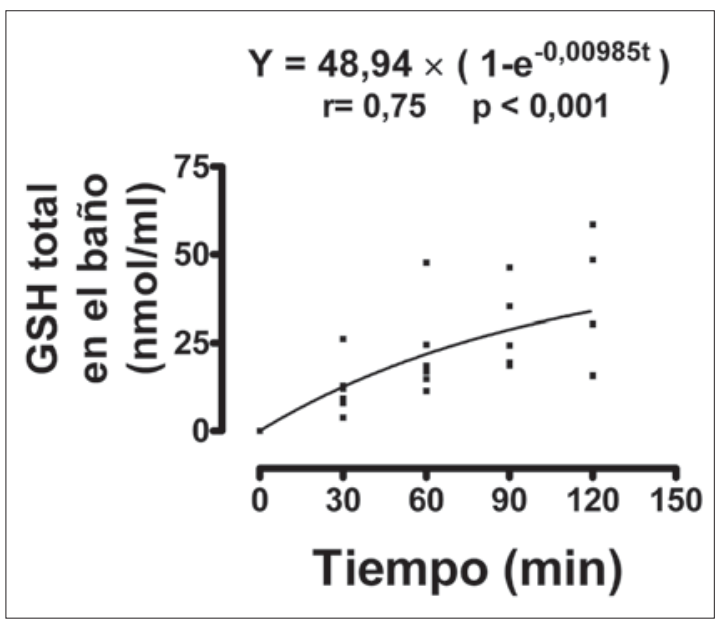

Figura 2. Aparición de glutatión y aumento progresivo de su concentración en la solución de preservación, que se ajusta a una curva exponencial $(r=0,75 ; \mathrm{p}<0,001)$. 


$$
\begin{gathered}
r=0,046 \\
p=0,9449
\end{gathered}
$$

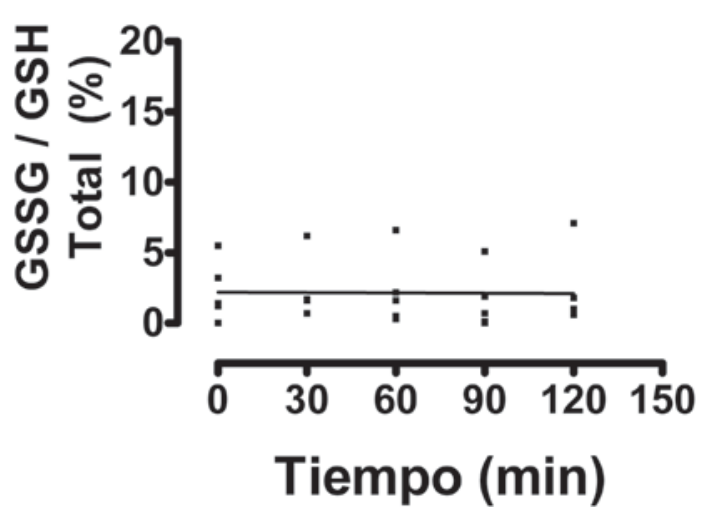

Figura 3. Concentración de GSSG expresado como equivalentes de glutatión reducido en porcentaje del GSH total, en el pulmón incubado en la solución de preservación fría. La línea continua representa la curva de regresión lineal con pendiente cercana a cero $(\mathrm{r}=0,46$; $\mathrm{p}<$ $0,9)$, que muestra que el contenido de GSSG no se modifica en el pulmón durante la incubación.

\section{Contenido de glutatión oxidado en pulmones a distintos tiempos de incubación en la solución de preservación para trasplante}

La Figura 3 muestra que el contenido de glutatión oxidado (GSSG), expresado como porcentaje del glutatión total, no se modificó en el pulmón incubado en la solución de preservación. El contenido de GSSG en la solución de preservación fue indetectable.

\section{Discusión}

Los resultados de este estudio muestran que en el pulmón de conejo mantenido en la solución de preservación que se utiliza para el tras- plante pulmonar en seres humanos, el contenido de glutatión disminuye progresivamente con el tiempo de incubación, mientras que este antioxidante aparece y aumenta progresivamente en la solución de preservación.

El sistema del glutatión es uno de los principales mecanismos antioxidantes del pulmón, ya que participa tanto a nivel celular como del líquido que cubre el epitelio alveolar ${ }^{16}$, protegiéndolo contra agentes oxidantes, compuestos electrofílicos y otros tóxicos. Forma parte de un ciclo de óxido-reducción ilustrado en la Figura 4, que es fundamental para prevenir la depleción de grupos -SH. En este ciclo, la reacción catalizada por la enzima glutatión peroxidasa (GPx) permite, a través de la oxidación del glutatión, la detoxificación del peróxido de hidrógeno y de distintos hidroperóxidos, mientras que la enzima glutatión reductasa (GRd), cataliza la reducción del glutatión oxidado (GSSG), el que se mantiene en niveles cercanos al $1 \%$ del glutatión total in vivo ${ }^{16}$.

Si bien la pérdida de antioxidantes desde tejidos conservados en soluciones de preservación para trasplantes ha sido descrita para órganos como el riñón, el corazón y el hígado ${ }^{9}$, existe muy poca información acerca de los mecanismos que explican dicha pérdida. El mecanismo propuesto por Vreugdenhil y cols ${ }^{9}$, para explicar la pérdida de GSH en los tejidos mencionados, dice relación con la posibilidad de que en condiciones de isquemia fría se inhiba la síntesis de GSH pero no su catabolismo. Es interesante destacar que si bien estos autores plantean la posibilidad de degradación del GSH, lo hacen sin haber medido la concentración de GSH ni de sus productos de degradación en la solución de preservación ${ }^{9}$.

Nuestro estudio muestra por primera vez, que una proporción importante del GSH que se pierde desde el pulmón se encuentra como tal y no

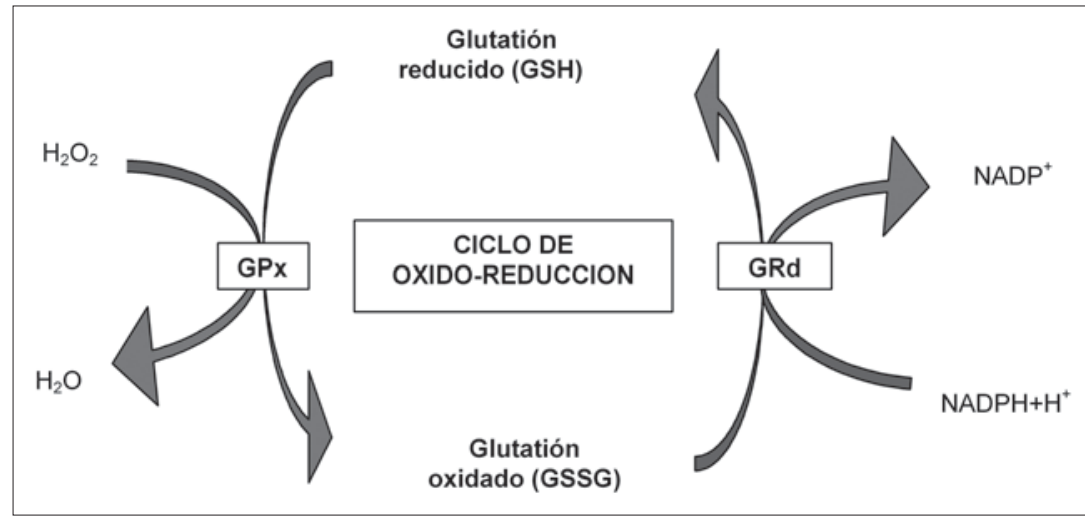

Figura 4. Ciclo de óxido-reducción del glutatión. GPx: Enzima glutatión peroxidasa. GRd: Enzima glutatión reductasa. 
como producto de degradación en la solución de preservación. Así, el paso de glutatión hacia la solución, fenómeno no descrito en la literatura para el pulmón, constituye uno de los mecanismos responsables de la disminución del GSH tisular.

Con respecto a la forma en que el glutatión sale del pulmón, es necesario considerar la exportación de la forma oxidada (GSSG), en condiciones en que su concentración aumenta debido a estrés oxidativo ${ }^{16}$. Sin embargo, en nuestro experimento, tanto el nivel basal de GSSG del pulmón como su concentración a lo largo del tiempo de incubación fueron muy bajos, cercanos a las concentraciones fisiológicas y lejos de la concentración requerida para activar mecanismos de exportación desde las células. Al mismo tiempo, no fue posible detectar GSSG en la solución de preservación. Por lo anterior, es posible afirmar que en ausencia de un aumento en la concentración de GSSG durante la mantención del pulmón en la solución de preservación, la pérdida de GSH desde el pulmón no se debe a salida de su forma oxidada. De igual forma, la mantención de los niveles de GSSG en rangos fisiológicos, hace poco probable que parte de la disminución del GSH tisular se explique por la participación del GSH en la formación de disulfuros mixtos con las proteínas tisulares, como ha sido descrito en condiciones de inflamación y estrés oxidativo ${ }^{17,18}$, las que no se dan en nuestro modelo que utiliza isquemia fría sin calentamiento posterior en conejos sanos. En la mayoría de los modelos experimentales de estrés oxidativo que muestran formación de disulfuros mixtos en los tejidos, éstos van acompañados de niveles altos de $\mathrm{GSSG}^{17,18}$. Planteamos que el GSH sale del tejido a favor del gradiente de concentración que se establece al incubar el pulmón que posee un alto contenido de GSH, en una solución que no contiene GSH. La forma exponencial de la curva de pérdida de GSH desde el tejido sumada a la forma también exponencial de la curva de aparición de GSH en el medio de incubación apoyan la existencia de un mecanismo favorecido por las diferencias de concentración.

Estudios futuros permitirán evaluar si el GSH encontrado en la solución de preservación da cuenta del total de la reducción en los niveles tisulares de este antioxidante.

Concluimos que existe pérdida de GSH desde el pulmón en condiciones de isquemia fría similares a las del trasplante pulmonar humano y que esta pérdida se acompaña de aparición de GSH en la solución de preservación. Dado que los niveles de GSSG del pulmón se mantuvieron normales, el mecanismo más probable es la salida de GSH en su forma reducida a favor del gradiente de concentración que se establece con la solución de preservación que no contiene GSH. Esta pérdida de GSH deja al pulmón en situación vulnerable frente a las múltiples condiciones que favorecen el estrés oxidativo en el receptor. Especulamos que el agregado de GSH a la solución de preservación podría, al disminuir la diferencia de concentración, prevenir la pérdida celular de GSH y mejorar el pronóstico del injerto.

\section{Bibliografía}

1.- PATTERSON G A. Indications. Unilateral, bilateral, heart-lung and lobar transplant procedures. Clin Chest Med 1997; 18: 225-30.

2.- TRULOCK E P, CHRISTIE J D, EDWARDS L B, BOUCEK M M, AURORA P, TAYLOR D O. et al. Registry of the International Society for Heart and Lung Transplantation: twentyfourth official adult lung and heart-lung transplantation report-2007. J Heart Lung Transplant. 2007; 26 (8): 782-95.

3.- PATEL N, DECAMP M, CRINER G J. Lung transplantation and lung volume reduction surgery versus transplantation in chronic obstructive pulmonary disease. Proc Am Thorac Soc 2008; 5: 447-53.

4.- HOSENPUD J D, BENNETT L E, KECK B M, FIOL $\mathrm{B}$, BOUCEK M M, NOVICK R J. The registry of the international society for heart and lung transplantation: sixteenth official report-1999. J Heart Lung Transplant 1999; 18: 611-26.

5.- FISCHER S, MATTE-MARTYN A, DE PERROT M, WADEELL T L, SEKINE Y, HUTCHE O N, et al. Low-potassium dextran preservation solution improves lung function after human lung transplantation. J Thorac Cardiovasc Surg 2001; 121: 594-6.

6.- PERROT M, KESHAVJEE S. Lung transplantation. Lung Preservation. Chest Surg Clin N Am 2003; 13: 443-62.

7.- KENNEDY $\mathrm{T}$ P, RAO $\mathrm{N}$ V, HOPKINS C, PENNINGTON L, TOLLEY E, HOIDAL J R. Role of reactive oxygen species in reperfusion injury of the rabbit lung. J Clin Invest 1989; 83: 1326-35.

8.- PERROT M, LIU M, WADDELL T K, KESHAVJEE S. Ischemia-reperfusion-induced lung injury. Am J Respir Crit Care Med 2003; 167: 490-511.

9.- VREUGDENHIL P K, BELZER F O, SOUTHLAND J H. Effect of cold storage on tissue and cellular glutathione. Cryobiology 1991; 28: 143-9.

10.- BORZONE G R, REYES T, RAMÍREZ B U. Glutathione content in the in vitro diaphragm preparation. Am J Respir Crit Care Med 151; A583, 1995. 
11.- SHIMOYAMA T, TABUCHI N, KOJIMA K, AKAMATSU $\mathrm{H}$, ARAI $\mathrm{H}$, TANAKA $\mathrm{H}$, et al. Aprotinin attenuated ischemia-reperfusion injury in an isolated rat lung model after 18-hour preservation. Eur J Cardiothorac Surg 2005; 28: 581-7.

12.- PADILLA A M, PADILLA J D. Lung preservation: current practices. Arch Bronconeumol 2004; 40: 8693.

13.- MACCHERINI M, KESHAVJEE S H, SLUTSKY A S, PATTERSON G A, EDELSON J D. The effect of low-potassium-dextran versus Euro-Collins solution for preservation of isolated type II pneumocytes. Transplantation 1991; 52: 621-6.

14.- GRIFFITH O W. Determination of glutathione and glutathione disulfide using glutathione reductase and 2-vinylpyridine. Anal Biochem 1980; 106: 207-12.
15.- BRADFORD M M. A rapid and sensitive method for the quantitation of microgram quantities of protein utilizing the principle of protein-dye binding. Anal Biochem 1976; 72: 248-54.

16.- RAHMAN I, MACNEE W. Oxidative stress and regulation of glutathione in lung inflammation. Eur Respir J 2000; 16: 534-54.

17.- RAHMAN I, Li X Y, DONALDSON K, HARRISON D J, MACNEE W. Glutathione homeostasis in alveolar epithelial cells in vitro and lung in vivo under oxidative stress. Am J Physiol Lung Cell Mol Physiol 1995; 269: L285-92.

18.- FORMAN H J, LIU R-M, TIAN L. Glutathione cycling in oxidative stress. en: Clerch LB, Massaro D eds. Oxygen, gene expression, and cellular function. New York, Marcel Dekker, Inc. 1977; 99-121.
Correspondencia a:

Dra. María Eliana Solovera R.

Sección de Cirugía de Tórax,

Pontificia Universidad Católica de Chile

Marcoleta 350 interior, piso 1, Santiago

Fono: 3546493

E-mail: solovera@med.puc.cl 